\title{
The Muscle-Mechanical Compromise Framework: Implications for the Scaling of Gait and Posture
}

\author{
by \\ James Richard Usherwood (Jim) ${ }^{1}$
}

\begin{abstract}
Many aspects of animal and human gait and posture cannot be predicted from purely mechanical work minimization or entirely based on optimizing muscle efficiency. Here, the Muscle-Mechanical Compromise Framework is introduced as a conceptual paradigm for considering the interactions and compromises between these two objectives. Current assumptions in implementing the Framework are presented. Implications of the compromise are discussed and related to the scaling of running mechanics and animal posture.
\end{abstract}

\section{Overview}

The purpose of this paper is to introduce and clearly delineate a new Framework for considering animal and human form and function, particularly in relation to locomotion. While the concepts underpinning the Framework have previously been applied to account for the scaling of a number of aspects of gait mechanics in walking, running and flapping flight, this paper introduces the Framework terminology, its application and implications more generally, with particular focus on the scaling of posture and running mechanics applicable to children and adult humans. Aspects of the history and development will be treated only briefly here, and the reader is encouraged to visit the primary literature for fuller recognition of previous work and more rigorous justification.

\section{The premise behind the Muscle- Mechanical Compromise Framework}

A bicycle allows vastly more economical locomotion than walking or running - cycling allows a mass or weight to be transported a given distance for far lower metabolic work. This can be attributed to two factors: the rolling wheel produces a low demand for mechanical work; and the pedals and adjustable gearing allow the mechanical work and power demands to be met by muscles operating in an economical manner with a high duty cycle (approximately 0.5 ) and at favourable stresses, strains and strain rates. Walking, running and flapping gaits cannot meet both of these demands for economical locomotion simultaneously; the two aspects - mechanical and muscle-physiological - have conflicting requirements.

Take running as an example. In terms of mechanical work alone, the most economical form of running is to contact the ground with very stiff legs and very brief stances (Srinivasan and Ruina, 2006); this minimizes the wasteful fore-aft accelerations and provides only the necessary impulses to give the ballistic aerial phase and time for the opposite leg to swing forward ready for the next stance. The problem is that this 'impulsive running' gait is highly unfavourable for muscle. While the total mechanical work is minimized, it remains non-zero as the body has to be propelled back upwards each step; and this work has to be performed in a near-zero time.

1 - Structure and Motion lab., The Royal Veterinary College, North Mymms, Hatfield, Herts, UK. 
This results in a huge (theoretically infinite) power demand during the stance. Given the majority of the power is produced directly by the muscle, this would require an enormous volume of muscle to be activated; and, given muscle activation is in itself physiologically costly, the gaits approaching the stiff-limbed, brief-stance impulsive running strategy would be metabolically highly uneconomical. Understanding why running - and indeed many animal gaits - achieves neither optimum economy in terms of mechanical work minimization, nor in terms of maximal muscle efficiency, requires an appreciation of the interplay between muscle and mechanical demands. The goal of developing the Muscle-Mechanical Compromise Framework (Figure 1) is to highlight this interplay, and offer a tractable route for including these interactions when interpreting animal form and function.

\section{When and how should the Framework be considered?}

The Framework may be of value whenever an aspect of form or function does not align with pure work minimization or conditions for maximal muscle efficiency - especially when selective pressures for metabolic economy might be expected to have been high and maintained. Clearly, this would include gaits used for long periods with high demands, such as migratory locomotion. But also, application may be found in cases of extreme performance - leaping, acceleration, maneuvering and maximal sprints of high selective value, but where muscular and mechanical demands are in conflict.

In order to apply the framework, the implications of a range of alternatives must be considered. In order to achieve this, a suitable, justifiable (and preferably mechanistic) range of alternatives or 'parameter space' should be modeled. The models must provide the implications of the parameter space in terms of a muscular demand (the cost function - presented here as dominated by activation) to provide the mechanical requirements (for instance, work and power over the contraction) of the kinematic, postural or morphological parameters of interest.

\section{Assumptions used in recent applications of the Framework}

The following assumptions are those developed for considering the scaling of animal posture (Usherwood, 2013), the scaling of walking and running mechanics with speed and size in children and adults (Hubel and Usherwood, 2015) and the scaling of bird flight strategies and gaits (Usherwood, 2016).

1. Metabolic cost of transport is the function being minimized. This appears a reasonable assumption generally supported by observation, at least for habitual gaits.

2. Metabolic cost is dominated by muscle activation cost. While this assumption is empirically well supported for steady level terrestrial locomotion (Kram and Taylor, 1990; Pontzer, 2007), it is clearly invalid in situations where large net work supply is demanded. It has been demonstrated recently that costs associated with work due to locomoting up an incline can be added to costs of activation to account for metabolic costs (Pontzer, 2016), but this has not yet been explored using the principles of the Framework.

3. Muscle activation is fundamentally demanded for (the more costly between) work and power during a contraction; mechanical gearing should be a sufficiently free parameter (through anatomy or posture) that 'force' activation costs (as distinct from work and power) can be neglected. This assumption prevents muscle force per se from being constraining or costly. While the forces applied to the muscles may correlate well with measured oxygen uptake, it is assumed within the Framework that only those forces are applied that load the muscle appropriately for their mechanical work and power demands. Note that this is a novel and somewhat controversial assumption, and limits the activities to be considered to habitual, energetically costly gaits.

4. Mechanical work and power demands during contraction can be modeled adequately assuming small deviations from idealized gaits. This allows the effects of mechanical demands (e.g. speed, size and details of gait such as stance duration) on the muscle to be calculated. The concept underlying the Framework focuses on the interactions and compromises between economical use of muscle and mechanical (workminimizing) economy. Despite advances in computer technology, attempting to model this interaction using detailed musculoskeletal models and dynamic optimization approaches without a 
guiding framework would result in a parameter space that is impractical. Consider for instance the greatly increased complexity associated with extending full forward dynamic simulation approaches (i.e. predictive simulations) to running where morphometric aspects (bone geometries etc.) are added to existing parameterizations of muscle activation profiles and kinematics as free parameters. In contrast, the approach adopted initially for use within the Framework, considering only small deviations from idealized gait mechanics, has the twin advantages of constraining the relevant parameter spaces and enabling intuitive interpretation of results.

5. Muscle properties are constant and muscles are used in a consistent manner. Specifically, work and power capacity are constant, as is their ratio (the 'Base muscle time', taken as 0.1 seconds). In detail, this assumption must be invalid, but it appears reasonable that muscle work and power capabilities (per muscle mass) scale less strongly than mechanical work and power demands (below).

\section{Notes on Supply, Demand and Interaction}

While the following presentation may appear 'supply-led', starting first with muscle, this is arbitrary. The Framework does not distinguish between 'supply-led' and 'demandled' viewpoints; both aspects are considered simultaneously.

\section{'Supply'}

Activating muscle has its own metabolic energy cost, even when the muscle is prevented from contracting and performs no mechanical work. This can be demonstrated by stimulating muscles held at a range of lengths (e.g. Homsher et al., 1972). When highly stretched, few crossbridges overlap and there is little tension; however, there remains a considerable energy cost which can be measured in vitro as a change in temperature.

Quite separate from the issues of activation costs, muscles can contract more or less efficiently to produce work; and different muscles can have different efficiencies. In general, 'slower' muscles are considered more efficient (Woledge, 1968) requiring a lower physiological energy supply to meet a mechanical work demand (Hill,
1950). However, contracting slowly produces little power, potentially meaning that gait mechanics are less economical and the mechanical work demands are higher. The Framework will allow this trade-off between muscle and mechanical economy to be explored, with the potential of revealing further implications of differences in muscle properties due to the fibre type, ageing, temperature, species or adaptation.

Base muscle time. This is a simple metric of considerable value within the Framework. It is the ratio of muscle work capacity (Joules/kg) to power capacity (Watts/kg), with dimensions of time. These capacities of a given muscle are those when the muscle is operating effectively in some way presumably at a high power or efficiency. For large warm-blooded vertebrates a value of $0.1 \mathrm{~s}$ has been adopted as an initial estimate (Hubel and Usherwood, 2015; Usherwood, 2013). This value would indicate that a contraction lasting less than $0.1 \mathrm{~s}$ demands a higher muscle activation to provide the power demands during the contraction than the mechanical work. To date, 0.1 $\mathrm{s}$ is merely a semi-empirical estimate, taken as invariant; at some level, this assumption must be untrue, potentially with revealing implications.

'Demand'

It is assumed that the demand for activation for a given set of gait parameters can be modeled by calculating the associated work and power requirements alone. By making the assumption that 'gearing' selected for a gait can be suitably selected to provide the best loading conditions for only the work and/or power demands, much of the system complexity can be reduced to a 'black box'. Cases can be imagined where this is invalid: for instance, consider the metabolic cost of supporting a load with an outstretched arm, even if it produces no net mechanical work or power; or the high metabolic cost of a chimpanzee walking bipedally (Pontzer et al., 2009). However, this assumption does appear reasonable for large terrestrial animals performing habitual locomotor tasks: gearing is simple to manipulate through an evolved form or with small adjustments of posture; why would animals 'choose' to load muscles with costly forces or velocities not fundamentally required by power and/or work?

Demand for mechanical work per se appears to have little bearing on the costs of 
steady, level locomotion for terrestrial animals over a large range of speeds and sizes. However, it clearly has cost implications during inclined locomotion. The interplay between minimizing the costs of activation and the physiological costs of performing mechanical work should be tractable within the Framework, and account for the longer stance duration adopted when running up-hill. The successful application of inverted pendulum walking mechanics to quantitatively predict reduction in the preferred walk-run transition with an incline (Hubel and Usherwood, 2013) demonstrates the value of considering small deviations from idealized work-minimizing gait mechanics.

\section{‘Supply-Demand Interaction'}

This is the key innovation of the Framework. In order to limit the parameter space of the potential interactions, and to allow mechanistically-based understanding, simplifying assumptions are required. To date, the focus has been on considering the implications of small deviations of gait kinetics from idealized workminimizing solutions (such as impulsive inverted pendulum walking, impulsive running and lowamplitude, steady flapping flight). An alternative that may be appropriate, particularly for small animals in which 'time' is very limited (of step, stance, swing or flap) is to consider small deviations from instantaneous power-minimizing gaits (see below).

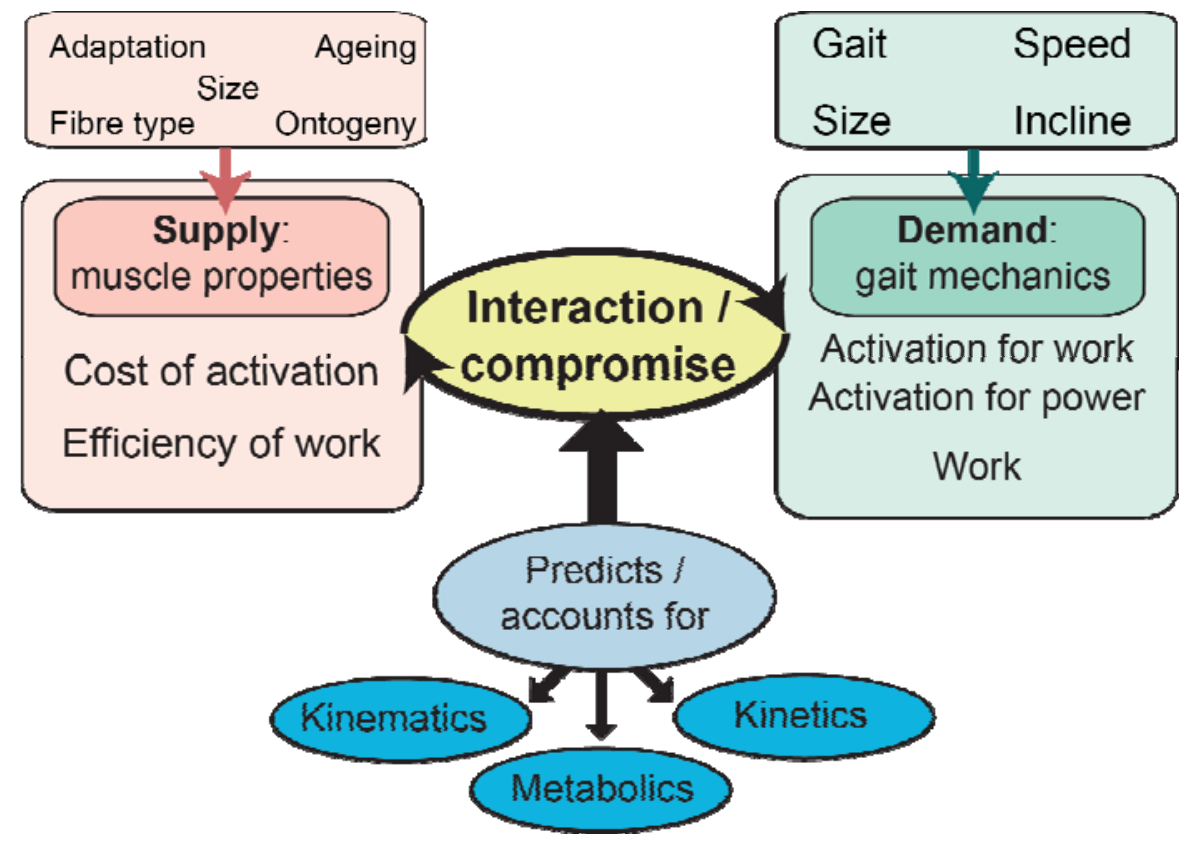

Figure 1

The Muscle-Mechanical Compromise Framework. To date, only the activation demands for steady, level gaits (walking, running, hopping and flapping

flight) have been considered within this Framework and the muscle properties

have been assumed to be largely constant. Future applications of the Framework will incorporate differences in muscle properties and additional mechanical demands,

especially the demands of net mechanical work while locomoting up inclines. 


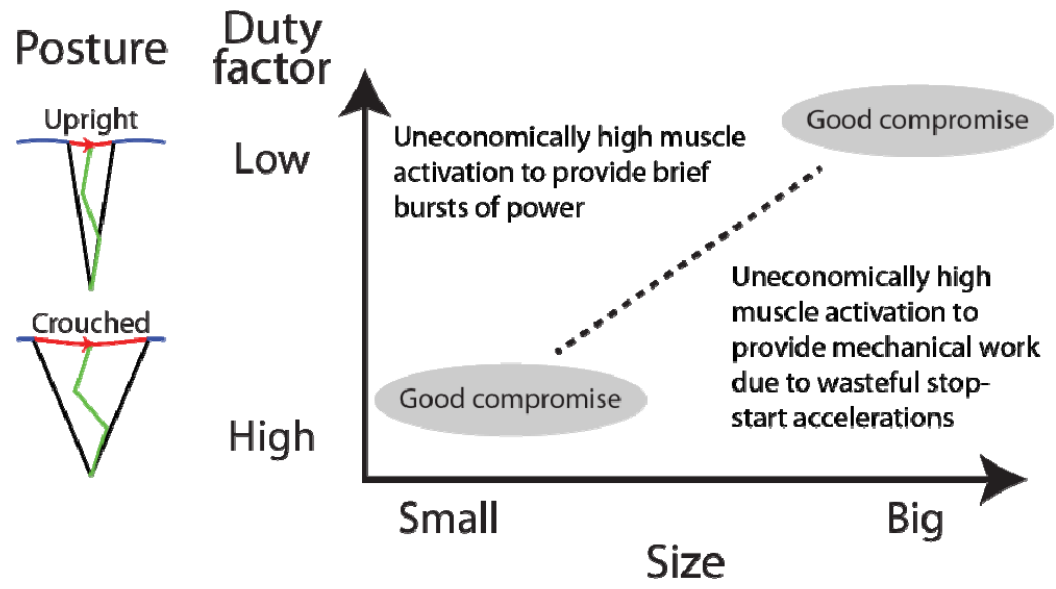

Figure 2

Diagrammatic representation of the implications of the Muscle-Mechanical

Compromise Framework as it relates to running gait mechanics

and the scaling of animal posture. The work and power demands on muscle contraction scale with size, such that large animals tend towards work minimization, with relatively brief stances and upright midstance posture. In contrast, smaller animals adopt strategies that ameliorate the muscle activation costs due to mechanical power demands during a stance, resulting in relatively high stance periods (duty factors), achieved with more crouched midstance posture.

\section{Findings from application of the Muscle-Mechanical Framework to running}

The gross mechanics of steady level running can be easily modeled given simple physical constraints - net weight support and no net fore-aft accelerations - and some assumptions concerning ground reaction force profiles that appear reasonably accurate, at least as a first approximation. Using such simple models to survey parameter spaces of leg length and stance duration shows or confirms that (Hubel and Usherwood, 2015):

1) Greater stance duration results in greater foreaft accelerations, and so greater work demanded by each stance.

2) Greater stance duration also (obviously) increases the duration over which this work can be applied, so the implications in terms of the required muscle contraction power are more complex. Increases in stance duration from very brief result in a decrease in contraction power demand due to the higher duration; however, at some stage increases in stance time result in such large increases in work that contraction power demands again increase, despite the increase in duration.

3) Dynamically similar running with small leg lengths results in disproportionately high contraction power demands (compared with work). Of course, this is not a new finding (Alexander and Jayes, 1983), but it may not be immediately intuitive and deserves a little expansion. While a mechanical cost of transport can be expressed in non-dimensional terms - as the mechanical work required per weight per distance - the work and power demands are dimensional and scale differently with leg length Lleg. This may be understood intuitively by 
considering a vertical leap. For a given weightspecific work capacity, all leapers should achieve the same height. However, jumping with short legs offers only a brief time in which to apply this work, and hence high power demands (BennetClark, 1977). The same applies to running: short legs are associated with brief stance periods (in absolute terms) and so muscles are challenged disproportionately by contraction power demands (versus work). For animals of different sizes locomoting with dynamic similarity, mechanical work demands scale in direct proportion to leg length, contrasting with power demands, which scale with $\sqrt{L_{\operatorname{leg}}}$. While some scaling of muscle properties is likely, this scaling often appears relatively weak (e.g. Seow and Ford, 1991) and is neglected in the initial applications of the Framework.

If we apply the preliminary cost model of the Framework - a muscle activation cost for whichever is more demanding between work and power during the contraction - to the mechanical relations for running described above, a number of phenomena can be explained. Figure 2 presents a qualitative expression of the findings.

\section{Why run with a foot-strike ahead of the body?}

'If your centre of gravity is behind your foot as it hits the ground... each footfall creates a braking effect. That's definitely not what you want' (Goater and Melvin, 2012, page 41). This, or very similar sentiments, can be found in very many books and magazines introducing running technique. While it appears mechanically intuitive - that any braking forces impede economical running and should be avoided - it fails to account for why even elite runners still produce braking forces each step when running at constant speed. Let us consider the implications of having the centre of mass directly over the foot as it (the foot) hits the ground. This could indeed result in zero braking effect; however, in order for fore-aft impulses to net zero - i.e. to run at a constant speed - the foot would also have to leave the ground after passing underneath the centre of mass... in other words, immediately. This would require the finite amount of work associated with changes of vertical velocity to be performed in zero time demanding infinite muscle activation to provide the contraction power. The Muscle-Mechanical
Compromise Framework therefore provides an account for why even elite athletes appear so wasteful: by investing in finite stance duration, despite the costs of increased mechanical work demand, the muscle activation costs due to contraction power can be reduced.

\section{Why do small children run with a very high duty factor?}

It is easily observed that children under the age of about 6 do not run in a dynamically similar manner to adults. Stance duration as a proportion of stride time (duty factor) is disproportionately long. Indeed, at suitably comparable speeds, their stance duration is approximately the same - in absolute terms - as that of adults (Hubel and Usherwood, 2015). While there may be numerous issues related to immature development of muscle, coordination and proportion, this observation can actually be understood as merely economical from the perspective provided by the Framework. The differential scaling of work and power means that the longer legged adults find muscle activation demanded by work disproportionately costly, driving relatively low contact times (low duty factors) and decreased work demands associated with fore-aft accelerations. If the shorter-legged children ran with the same duty factor as adults, they would experience excessively high muscle activation demands for the contraction power; hence, they use a higher duty factor, 'paying' more in terms of work due to fore-aft accelerations, in order to 'buy' more time in order to reduce the power demands.

\section{Why are small animals crouched (but large animals upright)?}

Answers to this question have previously focused on the differential scaling of weight (proportional to the cube of linear dimensions) to the capacity to support weight (proportional to the square of linear dimensions - the cross sectional area of the supporting structures). With this argument, larger animals are disproportionately challenged to support their weight and so evolve upright posture that supports the body weight more effectively (Biewener, 1989). However, this approach does not explain directly why smaller animals do not also take advantage of this upright posture, 
allowing a reduced investment and weight in supporting structures. The argument is usually that small animals are freer to make use of crouched postures, which presumably offer some advantage in terms of acceleration or maneuverability. An alternative account for the scaling of posture can be provided using the Muscle-Mechanical Framework. Furthermore, this different line of reasoning not only accounts for why large animals would benefit from being more upright, but also why small animals should be crouched. The argument here follows that described above: smaller animals find activation demands to provide contraction power relatively costly, whereas larger animals are more challenged by activation demands to produce work. If, then, smaller animals benefit from disproportionately long stance duration in order to ameliorate the power costs, this can be achieved with a highly flexed midstance leg position (Figure 2), which may account for the generally more crouched posture. While there is some support for this interspecifically among striding bipeds and quadrupeds (Usherwood, 2013), it does not appear to be true across bipedal hoppers (Gutmann et al., 2013). There currently appears to be no satisfactory and general mechanistic account for this contrast. Furthermore, it should be emphasized that, while an alternative account for the scaling of posture is possible within the Framework, the arguments underlying previous accounts remain very reasonable.

\section{Future directions}

Application of the Muscle-Mechanical Compromise Framework to date has been limited to highly simplified models of a steady, level gait and has relied on extreme assumptions concerning muscle properties and cost functions. The potential to extend the approach to include net mechanical work demands is currently being explored using incline locomotion. Generally, the challenge will be in adding appropriate and evidence-based levels of complexity - whether in terms of muscle parameters or gait mechanics while avoiding prohibitive computational demands or losing the potential for general insights.

\section{Acknowledgements}

This work (RVC manuscript reference CBS_01351) was funded by a Wellcome Trust Fellowship [095061/ Z/10/Z]. I am most grateful for the intellectual support provided by members of the Structure and Motion lab at the Royal Veterinary College.

\section{References}

Alexander R McN, Jayes AS. A dynamic similarity hypothesis for the gaits of quadrupedal mammals. J Zool, 1983; 201: 135-152

Bennet-Clark HC. Scale effects in jumping animals. In Scale effects in animal locomotion (ed. TJ Pedley). London, UK:Academic Press; 185 - 201; 1977

Biewener AA. Scaling body support in mammals: limb posture and muscle mechanics. Science, 1989: 245; 4548

Goater J, Melvin D. The art of running faster. Human Kinetics. ISBN-13: 978-0-7360-9550-1; 2012

Gutmann AK, Lee DV, McGowan CP. Collision-based mechanics of bipedal hopping. Biol Lett, 2013; 9. Doi: 10.1098/rsbl.2013.0418

Hill AV. The dimensions of animals and their muscular dynamics. Science Progress, 1950; 38: 209-230

Homsher E, Mommaerts WFHM, Ricciuti NV, Wallner A. Activation heat, activation metabolism and tension-related heat in frog semitendinosus muscles. J Physiol, 1972; 220: 601-625

Hubel TY, Usherwood JR. Vaulting mechanics successfully predict decrease in walk-run transition speed with incline. Biol Lett, 2013; 9. doi: 10.1098/rsbl.2012.1121.

Hubel TY, Usherwood JR. Children and adults minimise activated muscle volume by selecting gait parameters that balance gross mechanical power and work demands. J Exp Biol, 2015; 218: 2830-2839 
Kram R, Taylor CR. Energetics of running: a new perspective. Nature, 1990; 346: 265-267

Pontzer H. Predicting the energy cost of terrestrial locomotion: a test of the LiMb model in humans and quadrupeds. J Exp Biol, 2007; 210: 484-494

Pontzer H, Raichlen DA, Sockol MD. The metabolic cost of walking in humans, chimpanzees, and early hominins. J Hum Evol, 2009; 56: 43-54

Pontzer H. A unified theory for the energy cost of legged locomotion. Biol Lett, 2016; DOI: 10.1098/rsbl.2015.0935

Seow CY, Ford LE. Shortening velocity and power output of skinned muscle fibers from mammals having a 25,000-fold range of body mass. J Gen Physiol 1991; 97: 541-560

Srinivasan M, Ruina A. Computer optimization of a minimal biped model discovers walking and running. Nature, 2006; 439: 72-75

Usherwood JR. Constraints on muscle performance provide a novel explanation for the scaling of posture in terrestrial animals. Biol Lett, 2013; 9. Doi: 10.1098/rsbl.2013.0414

Usherwood JR. Physiological, aerodynamic and geometric constraints of flapping account for bird gaits, and bounding and flap-gliding flight strategies. J Theor Biol, 2016; 408: 42-52. Doi: 10.1016/j.jtbi.2016.07.003

Woledge RC. The energetics of tortoise muscle. J Physiol, 1968; 197: 685-707

\section{Corresponding author:}

\section{James Richard Usherwood (Jim)}

Structure and Motion lab., The Royal Veterinary College

North Mymms, Hatfield, Herts, AL9 7TA, UK

E-mail: jusherwood@rvc.ac.uk 